\title{
Mapping of Enhanced Nuclear Stability in the Heaviest Elements
}

K. J. Moody, J. F. Wild, N. J. Stoyer, M. A. Stoyer, C. A. Laue, R. W. Lougheed

\section{March 6, 2001}

Lawrence

Livermore

National

Laboratory 


\section{DISCLAIMER}

This document was prepared as an account of work sponsored by an agency of the United States Government. Neither the United States Government nor the University of California nor any of their employees, makes any warranty, express or implied, or assumes any legal liability or responsibility for the accuracy, completeness, or usefulness of any information, apparatus, product, or process disclosed, or represents that its use would not infringe privately owned rights. Reference herein to any specific commercial product, process, or service by trade name, trademark, manufacturer, or otherwise, does not necessarily constitute or imply its endorsement, recommendation, or favoring by the United States Government or the University of California. The views and opinions of authors expressed herein do not necessarily state or reflect those of the United States Government or the University of California, and shall not be used for advertising or product endorsement purposes.

This work was performed under the auspices of the U.S. Department of Energy by the University of California, Lawrence Livermore National Laboratory under Contract No. W-7405-Eng-48.

This report has been reproduced directly from the best available copy.

Available electronically at http://www.doc.gov/bridge

Available for a processing fee to U.S. Department of Energy And its contractors in paper from

U.S. Department of Energy

Office of Scientific and Technical Information P.O. Box 62

Oak Ridge, TN 37831-0062

Telephone: (865) 576-8401

Facsimile: (865) 576-5728

E-mail: reports@adonis.osti.gov

Available for the sale to the public from

U.S. Department of Commerce

National Technical Information Service

5285 Port Royal Road

Springfield, VA 22161

Telephone: (800) 553-6847

Facsimile: (703) 605-6900

E-mail: orders@ntis.fedworld.gov

Online ordering: http://www.ntis.gov/ordering.htm

OR

Lawrence Livermore National Laboratory

Technical Information Department's Digital Library

http://www.Ilnl.gov/tid/Library.html 


\section{LDRD final report}

Mapping of Enhanced Nuclear Stability in the Heaviest Elements

K. J. Moody, J. F. Wild, N. J. Stoyer, M. A. Stoyer, C. A. Laue, R. W. Lougheed 98-ERD-050

Predictions of the properties of nuclides near the extreme limits of nuclear stability provide a measure of our understanding of the fundamental properties of matter and the fission process. Predictions of an "island of stability" of long-lived superheavy elements beyond the limits of the known nuclides date back more than 30 years; during this time, there have been many unsuccessful searches for these nuclei. During the last decade, there has been a large effort by our group and others to systematically discover and characterize the properties of the intervening unstable nuclei. Starting 10 years ago, in an on-going collaboration with Russian scientists at the Joint Institute for Nuclear Research (JINR) at Dubna, Russia, we observed the decays of previously unknown isotopes of elements 106,108 , and 110 whose properties are determined by subtleties in the nuclear structure caused by the shell effects that are predicted to result in the island of stability in the still-heavier elements. The resulting data have been successfully reproduced by the theoreticians, whose refined predictions of the decay modes and production rates of the superheavy elements have enabled us to design experiments with the sensitivity to locate these elusive nuclides.

For LDRD project 98-ERD-050, we continued our collaboration with the Russian scientists at JINR. We performed experiments to discover and characterize the superheavy isotopes around the magic nucleon numbers $\mathrm{Z}=114$ and $\mathrm{N}=184$, produced in the bombardment of ${ }^{244} \mathrm{Pu}$ and ${ }^{248} \mathrm{Cm}$ targets with ${ }^{48} \mathrm{Ca}$ ions. The most neutron-rich isotopes of superheavy elements and, consequently, the most stable against spontaneous fission (SF) decays, are expected to be produced in the reactions of actinide targets with the doubly-magic ${ }^{48} \mathrm{Ca}$ projectile.

A beam of $0.7 \mathrm{p \mu A}-{ }^{48} \mathrm{Ca}$ ions was delivered by the $\mathrm{U} 400$ cyclotron to $32-\mathrm{cm}^{2}$ rotating targets consisting of the enriched isotopes ${ }^{244} \mathrm{Pu}(98.6 \%)$ and ${ }^{248} \mathrm{Cm}(96.3 \%)$. Evaporation residues (EVRs) recoiling from the targets were separated in flight from beam particles, scattered ions and various transfer-reaction products by the Dubna Gas-filled Recoil Separator (fig. 1), passed through a time-of-flight (TOF) detector, and were implanted in focal-plane detectors, consisting of twelve $4-\mathrm{cm}$-high $\times 1-\mathrm{cm}$-wide position-sensitive strips. To detect $\alpha$ 's escaping the focal-plane detector, eight side detectors of the same type but without position sensitivity were arranged in a box surrounding the focal-plane detector. The total efficiency for detecting $\alpha-$ particles with full energy was $\sim 87 \%$ of $4 \pi$. A set of 3 similar "veto" detectors was situated behind the focalplane detectors in order to eliminate signals from low-ionizing light particles, which could pass through the focal-plane detector ( $300 \mu \mathrm{m}$ thick) without being detected in the TOF system. With a continuous ${ }^{48} \mathrm{Ca}$ beam with an intensity of $4 \times 10^{12} \mathrm{pps}$, the overall counting rate of the detector system was $\sim 15 \mathrm{~s}^{-1}$. We estimate from calibration reactions that $40 \%$ of the recoiling $\mathrm{Z}=114$ nuclei formed in the ${ }^{244} \mathrm{Pu}$ target would be implanted in the focal-plane detector.

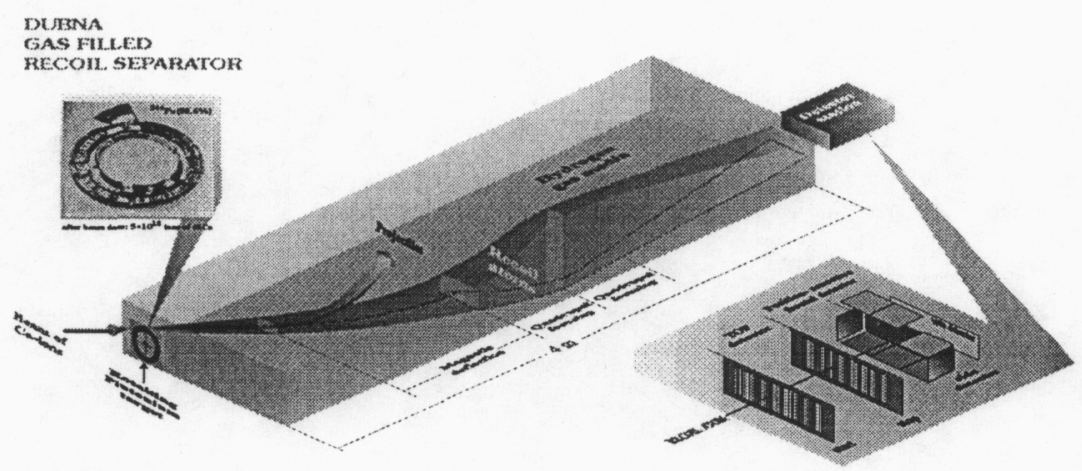

Figure 1. Schematic diagram of the Dubna Gas-filled Separator 
Alpha-energy calibrations were periodically performed using the $\alpha$ peaks from nuclides produced in the test reaction ${ }^{206} \mathrm{~Pb}+{ }^{48} \mathrm{Ca}$. The fission-energy calibration was obtained by detecting fission fragments from the $\mathrm{SF}$ of ${ }^{252} \mathrm{No}$. The energy resolution for detection of $\alpha$-particles in the focal-plane detector was $\sim 50 \mathrm{keV}$; for detection by the side detectors of $\alpha$ 's escaping from the focal-plane detector, the energy resolution was $-190 \mathrm{keV}$. We determined the position resolution of the signals of correlated decays of nuclei implanted in the detectors: Typically, for sequential $\alpha-\alpha$ decays the FWHM position resolution was approximately $1.1 \mathrm{~mm}$; for correlated EVR- $\alpha$ signals, approximately $0.8 \mathrm{~mm}$; and for correlated EVR-SF signals, approximately $0.5 \mathrm{~mm}$.

The characteristic decay sequence of a superheavy nucleus has been predicted to be a series of alpha decays, terminated by a spontaneous fission. Because of the efficiency of the separator for suppressing unwanted reaction products, detected SF events were rare; we observed only about thirty of them over the course of our experiments, most of them during the irradiation of ${ }^{248} \mathrm{Cm}$ targets. The origin of most of these $\mathrm{SF}$ events is $\mathrm{Cf}$ and $\mathrm{Fm}$ isotopes incompletely filtered out by the separator, whose suppression efficiency is about $10^{5}$. We searched the data backwards in time from these events for preceding $\alpha$ particles with $E_{\alpha}>8 \mathrm{MeV}$ and/or EVRs, in the same positions. The latter were defined as events characterized by the measured energies, TOF signals and estimated resulting mass values that were consistent with those expected for a complete-fusion EVR, as determined in the calibration reactions. Many of the detected SF events were preceded immediately by an EVR, and are explainable by fission isomers that weren't completely removed by the separator. Five events were position-correlated with EVRs with at least two intervening alpha events.

One event sequence (see figure 2), observed in the bombardment of ${ }^{244} \mathrm{Pu}$ with ${ }^{48} \mathrm{Ca}$, we have attributed to the mass-289 isotope of element 114; an EVR event is followed by three alpha and a spontaneous fission, strongly correlated in position, which occurred over a span of 30 minutes. The decay sequence, started by the initial alpha emission by ${ }^{289} 114$, which decays with an amazingly long $30-$ s lifetime, charts the progress of the decay away from the island of stability into species that are unstable to SF decay. A Monte Carlo technique was employed that determined the chances of this multiple correlation in time and position being due to random events is very small.

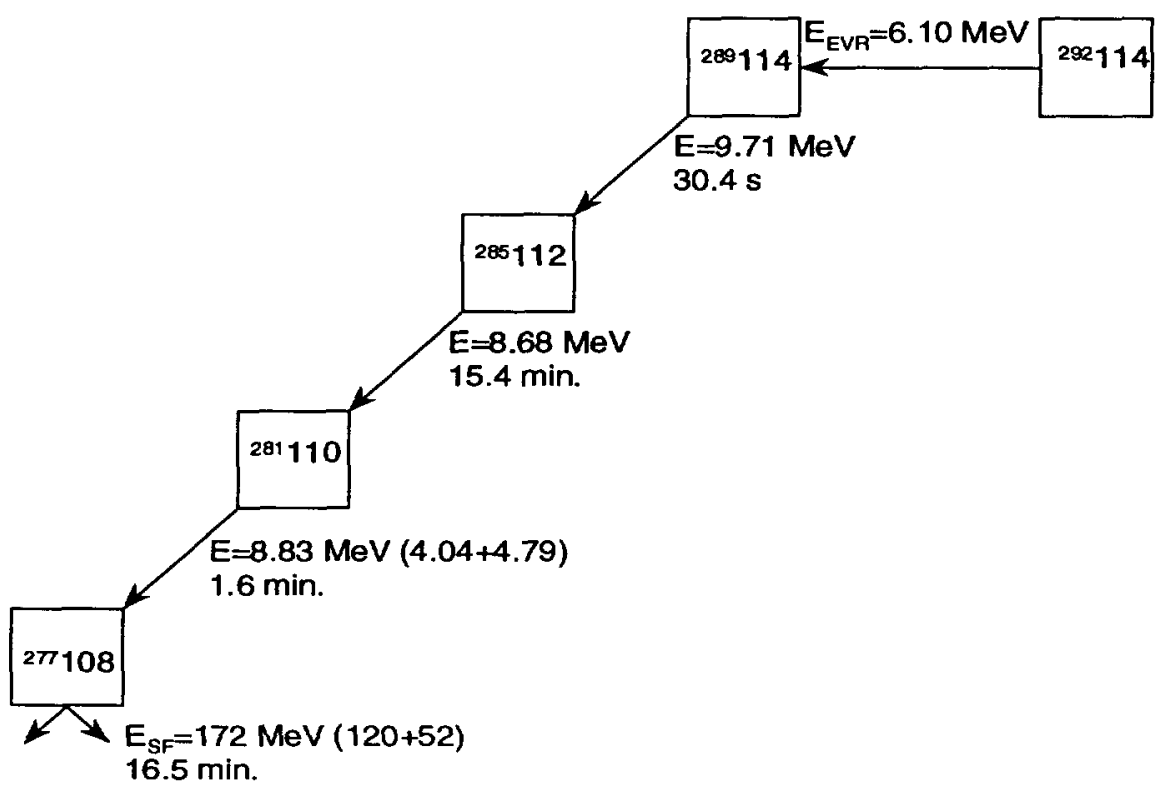

Figure 2. The decay of a single atom of ${ }^{289} 114$ 
Also in the bombardment of ${ }^{244} \mathrm{Pu}$, we observed two similar decay sequences that we attribute to the decay of two atoms of ${ }^{288} 114$, resulting from the evaporation of four neutrons from the compound nucleus The full decay chains including these two fission events are shown in Fig. 3b, with the suggested assignment of decays to specific nuclei. The chance of either chain being of random origin is small; the chances that two such similar chains would arise randomly in the data are insignificant.

We started an experiment designed to synthesize superheavy nuclei with $\mathrm{Z}=116$ in the reaction ${ }^{248} \mathrm{Cm}+$ ${ }^{48} \mathrm{Ca}$. In a 91-day bombardment, after a beam dose of $6.6 \times 10^{18}{ }^{48} \mathrm{Ca}$ ions, we observed a decay chain consisting of three consecutive $\alpha$-decays and an SF. Implantation of an EVR in the focal-plane detector was followed $47 \mathrm{~ms}$ later by an $\alpha$-particle decay with $E_{\alpha}=10.56 \mathrm{MeV}$. This sequence switched the ion beam off, and further decays were detected under low-background conditions. The energies and decay times of the descendant nuclei (see figure 3a) are in agreement with those observed in the decay chains of the even-even isotope ${ }^{288} 114$ (see figure $3 b$ ). Thus, the first $\alpha$-decay should be attributed to the parent nuclide ${ }^{292} 116$, produced via the evaporation of four neutrons in the complete-fusion reaction ${ }^{248} \mathrm{Cm}+{ }^{48} \mathrm{Ca}$. All the decay chain members follow the Geiger-Nuttal $E_{\alpha} v s T_{\alpha}$ relationship for even-even nuclei. Substituting the values $E_{\alpha}=9.83 \pm 0.05 \mathrm{MeV}$ and $\mathrm{T}_{\alpha}=1.8^{+2.1}-0.6 \mathrm{sec}$ (the properties assigned to ${ }^{288} 114$ based on the three decay chains) into the Geiger-Nuttal expression results in the atomic number $114.3 \pm$ 1.0 .

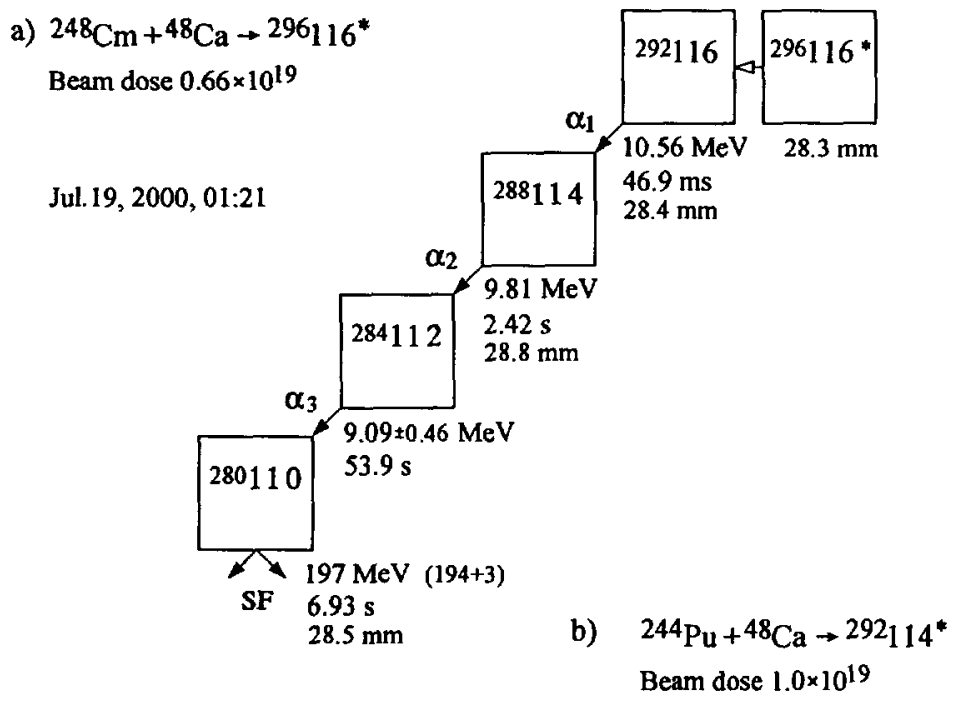

Fig. 3 a) Time sequence in the observed decay chain assigned to ${ }^{292} 116$, produced in the reaction of ${ }^{248} \mathrm{Cm}$ with ${ }^{48} \mathrm{Ca}$.

b) Two decay sequences of ${ }^{288} 114$ observed in the ${ }^{244} \mathrm{Pu}+{ }^{48} \mathrm{Ca}$ reaction. Vertical positions of the observed events are given with respect to the top of the strip. Values in parentheses show fission energies measured by the focal-plane and side detectors, respectively. 
The fifth SF event position correlated with alpha decays, also observed in the ${ }^{48} \mathrm{Ca}+{ }^{248} \mathrm{Cm}$ run, is still being interpreted.

The radioactive decay properties of the even-even 114 and 116 isotopes are in qualitative agreement with macroscopic-microscopic nuclear theory, which predicts both $\alpha$-decay and SF properties of the heavy nuclei. Alpha-decay energies of the synthesized nuclide and those of previously known isotopes of even- $Z$ elements with $Z \geq 100$, together with theoretical $Q_{\alpha}$ values for even-even isotopes of the $Z=106-116$ elements, are shown in Figure 4. It can be seen that the $\alpha$-decay energy of the new even-even nuclide ${ }^{292} 116$, as well as those of other heaviest even-even nuclei with $\mathrm{Z}=112$ and 114 , are 0.35 to $0.5 \mathrm{MeV}$ less than the corresponding predicted values. Such a decrease in $Q_{\alpha}$ values leads to an increase of partial $\alpha$ decay lifetimes by an order of magnitude. Thus, the comparison of the measured decay properties of the superheavy nuclei with theoretical calculations confirm the supposition that nuclei in the vicinity of spherical shell closures with $Z=114$ and $N=184$ could be even more stable than is predicted by theory.

Along with the observation of a third element-114 isotope by another Dubna group, and still another isotope of element 114 as part of the decay chain of the recent element-118 discovery by the Lawrence Berkeley National Laboratory group, we feel we have made an excellent start at mapping the extent and strength of the nuclear shell effect that results in the island of stability.

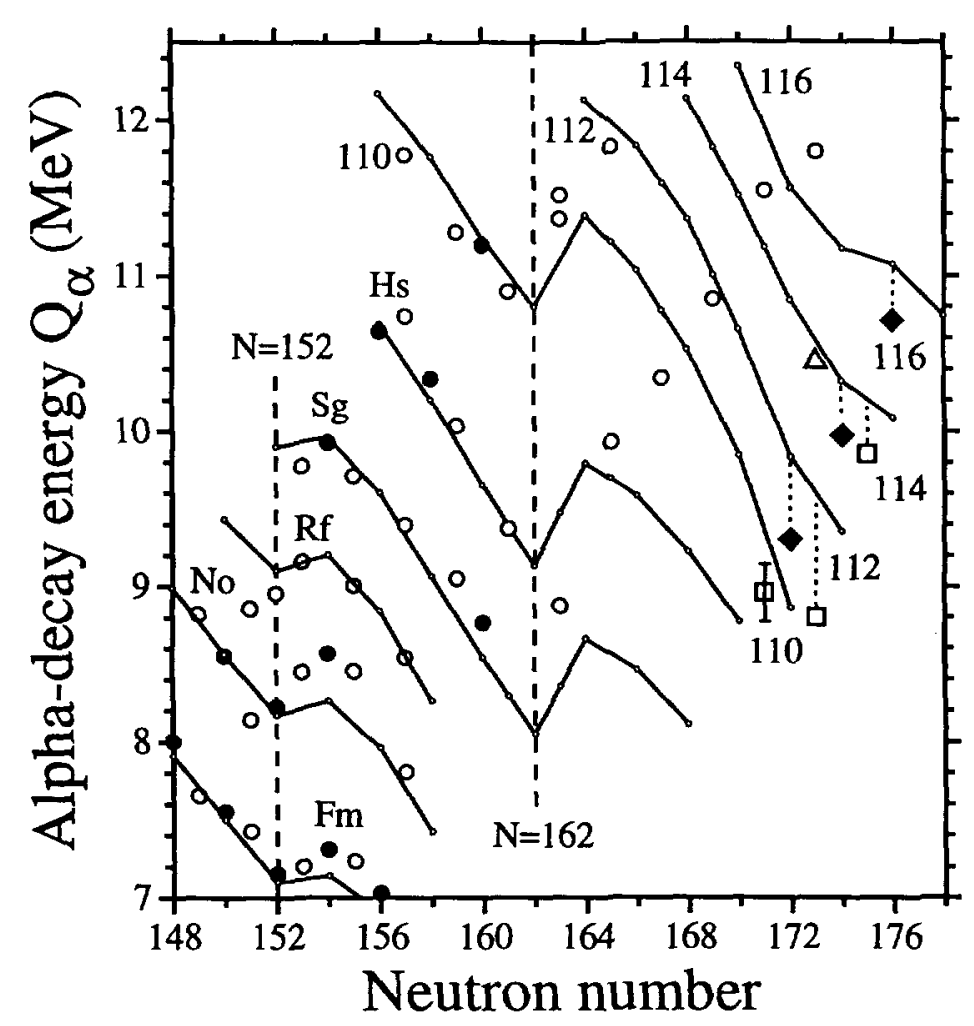

Fig. 4. Alpha-decay energy vs. neutron number for isotopes of even- $Z$ elements with $Z \geq 100$ (solid circles even-even isotopes, open circles - even-odd isotopes) [17-25]. Squares and diamonds denote data from the present work. Open circles connected with solid lines show theoretical $Q_{\alpha}$ values [7,8] for even-even $Z=100-116$ isotopes. 